\title{
Prescripciones alimentarias y límites de la medicalización: polifonía y utilización de medios de comunicación en una población urbana de México
}

\author{
Food prescriptions and limits of medicalization: \\ polyphony and use of the media in an urban \\ population of Mexico
}

José Alejandro Meza-Palmeros

(D) https://orcid.org/0000-0003-2424-7879

E-mail: alejandro.mezaळciesas.edu. $\mathrm{mx}$

Centro de Investigaciones y Estudios Superiores en Antropología Social. Monterrey, NL, México.

\section{Correspondencia}

Calle José María Morelos, 822. Monterrey, NL, México. CEP 64000.

\section{Resumen}

La línea argumental del presente trabajo sostiene que la polifonía discursiva contenida en la información relacionada con prescripciones alimentarias, que reproducen diversos sujetos discursivos, contribuye a generar resignificaciones en la población sobre las prescripciones propuestas que se traducen en prácticas alimentarias de refracción, las mismas que pudieran explicar el impacto limitado que han tenido las intervenciones educativas para la promoción de “estilos de vida saludables". El objetivo fue estudiar el proceso de refracción de la información prescriptiva sobre alimentación e identificar las prácticas alimentarias generadas en la población det un barrio de Monterrey, México, a partir de su interacción con sujetos discursivos claves, incluidos los medios de comunicación. Se realizó una etnografía polifónica de diez meses de duración que incluyó observación participante, entrevistas etnográficas y una encuesta. Se identificaron tres tipos de discursos prescriptivos: restrictivo, selectivo y asociado a la medicamentación. Producto de la refracción de dichos discursos, la población ha generado prácticas de sustitución y de restricción de ciertos alimentos, manifestando con ello su preocupación por la salud infantil.

Palabras clave: Etnografía; Educación para la Salud; Medios de Comunicación Social; Infancia; Políticas de Salud. 
The argumentative approach of this work sustains that the "polyphony" speech integrated in the information associated with food, contributes to generate a community resignification regarding the proposed prescriptions by several discursive subjects, which are interpreted into "refractive feeding practices." These could explain the limited impact that educational interventions have had to the "healthy lifestyles" promotion. The purpose was to study the refractive process related to a prescriptive information about nutrition and identifying the feeding practices generated precisely on a Monterrey, Nuevo León settlement, from their interaction with a discursive framework on key subjects, including the media. A polyphonic ethnography was conducted in ten months, and it included participant observation, ethnographical interviews and a survey. Three prescriptive discourses were identified: restrictive, selective and one associated with "medicamentation." As a result of refraction of the mentioned discourses, the population generated substitution practices and a restriction on certain food products, expressing their concern for children's health. Keywords: Ethnography; Health Education; Social Media; Childhood; Health Policy.
La educación para la salud constituye una de las principales políticas en salud para atender el problema de la obesidad infantil. La intervención del Estado para prescribir prácticas alimentarias acorde con el discurso biomédico permite plantear el problema como medicalización.

Sin embargo, es importante reconocer que en la actualidad la población recibe información diversa, contradictoria y de manera continua a través de los medios de comunicación, además de la información proveniente de instancias sanitarias (Carpenter et al., 2016), lo que destaca el carácter polifónico de la medicalización, cuyos efectos en la población deben ser estudiados, aspecto que constituye una parte sustantiva del presente trabajo.

La educación para la salud ha mostrado tener un impacto limitado e inconstante, particularmente en el caso de la obesidad infantil (Loveman et al., 2015). Distintas evaluaciones realizadas en México muestran resultados similares (Batis et al., 2016; Safdie et al., 2014), aunque, de acuerdo con la última encuesta de salud y nutrición, se reportó una disminución del problema en el norte del país (INSP; SSA, 2016). Las discrepancias encontradas expresan la existencia de una adopción diferenciada de prescripciones alimentarias y de procesos heterogéneos de medicalización.

Una hipótesis que puede explicar esta heterogeneidad radica en el carácter polifónico de la reproducción del discurso biomédico. Esta polifonía, como lo argumentaré, implica diversos procesos de significación y, en consecuencia, la generación de "prácticas alimentarias de refracción". Aunque estas expresan la preocupación de los sujetos y la puesta en práctica de medidas para mejorar y cuidar su salud, no necesariamente son las esperadas por las autoridades sanitarias.

La aproximación polifónica al estudio del proceso de salud, enfermedad, atención, en particular en su dimensión semiótica, puede contribuir a comprender los procesos de medicalización en general. Los hallazgos empíricos, por su parte, permitirán valorar los efectos que determinadas intervenciones en salud tienen sobre la población, sobre todo al integrar al análisis a los medios de 
comunicación, cuya capacidad para la producción de representaciones de la salud y la enfermedad es fundamental (Gómez; Díaz, 2019).

\section{Medicalización y polifonía}

La medicalización alude a la influencia que la biomedicina ejerce en (1) la definición de objetos de intervención; (2) la construcción de representaciones sociales de la salud y enfermedad (Conrad, 1992) y (3) la creación de dispositivos que posibilitan la realización de prescripciones sobre padecimientos, cuerpo y vida cotidiana (Rose, 2007).

La educación para salud, como señala Rosen (1985), ha sido desde el siglo XIX uno de los principales dispositivos de la medicina de Estado para la construcción de objetos de intervención.

Trabajos recientes han permitido apreciar la extensión y diversidad de los dispositivos de medicalización, al identificar sujetos discursivos que mantienen autonomía respecto de la institución médica, tales como la industria farmacéutica (Conrad, 1992), las organizaciones no gubernamentales, las medicinas alternativas (Domínguez, 2019) y los medios de comunicación (Briggs, 2003).

Esta multiplicidad de sujetos que intervienen en el proceso de medicalización desde distintas posiciones, intereses e interpretaciones, invita a pensar en clave de polifonía, según Bajtín (1982). ${ }^{1}$ Para este trabajo considero "sujetos discursivos" a los agentes que reproducen un determinado discurso y, por "dispositivo", me refiero al conjunto heterogéneo de prácticas discursivas y no discursivas (instituciones, saberes y medios) cuyo objetivo es la obtención de un efecto intencionado en los comportamientos (Agamben, 2011).

\section{Prácticas alimentarias de refracción}

Algunos estudios han mostrado la relevancia que los procesos de significación tienen en la adopción de prácticas alimentarias (Arcan et al., 2018). Sin embargo, salvo excepciones como el trabajo de Théodore et al. (2011), el discurso biomédico no es analizado, a pesar de que este se encuentra extendido en amplios sectores de la población. Por otra parte, los significados se han estudiado de manera independiente de las condiciones materiales de existencia, a pesar de que estas son determinantes (Drewnowski, 2012).

En este trabajo retomo el concepto de "refracción" (Volóshinov, 2009), considerándola como la articulación y resignificación de información polifónica, en un determinado contexto material de vida.

El objetivo del presente trabajo es estudiar el proceso de refracción de la información prescriptiva sobre alimentación e identificar las prácticas alimentarias generadas en la población de un barrio de Monterrey, México, a partir de su interacción con sujetos discursivos clave. Los hallazgos son producto de una investigación realizada mediante una etnografía realizada de enero a octubre de 2019.

\section{Material y métodos}

La zona de estudio fue elegida por la oportunidad de entrar en contacto con una organización que ahí interviene. Una de las actividades realizadas por esta organización es una capacitación periódica denominada "minichef", dirigida a niños de entre 5 y 12 años. La finalidad es fomentar la autonomía e independencia mediante la impartición de conocimientos de cocina, ya que muchos de ellos permanecen solos en casa mientras sus padres trabajan. Dado que las capacitaciones son llevadas a cabo por mujeres residentes de la zona, consideré que era un espacio ideal de observación, ya que se reproducirían valoraciones y significados locales otorgados a los alimentos. Logré involucrarme como observador-participante durante 20 sesiones.

Apliqué una encuesta ( $\mathrm{n}=60$ viviendas) como punto de partida para conocer el acceso y utilización de medios de comunicación de la población.

Paralelamente participé como facilitador en 10 talleres de promoción de estilos de vida saludables solicitados por la Dirección de Prevención del Delito

1 Coexistencia de "un determinado conjunto de ideas, pensamientos y palabras que se conduce a través de varias voces separadas, sonando cada una de ellas de manera diferente” (Bajtín, 1982, p. 190). 
de la policía municipal de Monterrey, que desarrolla un programa denominado "Regios Trabajando", cuya actividad principal es la "recuperación del espacio público".

A los talleres acudieron 12 mujeres cuyas edades oscilan entre 30 y 65 años y poseen una característica en común: fungen como cuidadoras de infantes, ya sea como madres (tres) o como abuelas (nueve), característica que las involucra en la alimentación de la población infantil y en la adquisición de alimentos. Parte de su cotidiano alimentario consiste en un consumo considerable de productos altos en azúcares y harinas refinadas. Esto refleja la poca disponibilidad de vegetales en la zona.

Durante los talleres se indujo una discusión siguiendo tres ejes temáticos: efecto de la televisión y de las redes sociales en sus prácticas alimentarias; valoraciones sobre lo saludable en los alimentos y prácticas llevadas a cabo de acuerdo con dichas valoraciones.

Estas actividades me ayudaron a ingresar al barrio y convivir con una familia, involucrándome en algunas de sus actividades cotidianas de compra, cocina y consumo de alimentos, incluso en el acompañamiento de una de las abuelas a consulta médica para realizar el seguimiento de su diabetes. También asistí, junto con ella, a "reuniones de alimentación" organizadas por una empresa que vende productos nutricionales en la zona.

Además de las acciones descritas participé como observador en seis talleres para la promoción de estilos de vida saludables, tres de los cuales fueron organizados por empresas privadas; uno por el Instituto Estatal de las Mujeres y dos más por la Secretaría de Salud. Visité 4o centros de salud como usuario simulado (Meneses-Navarro; Meléndez-Navarro; Meza-Palmeros, 2018), para conversar con promotores de salud y nutriólogos.

Realicé 26 entrevistas etnográficas, caracterizadas por la situación informal en que ocurrieron y por su no directividad (Guber, 2001). De estas, cinco fueron con mujeres residentes de la zona, cinco con interventores (dos miembros de ONG, dos policías y un médico) y 16 con actores clave en la reproducción del discurso biomédico público (nueve promotoras de salud, tres nutriólogas, dos coordinadores y dos directivos de programas Estatales relacionados con educación en salud).

Toda la información obtenida a través de mis observaciones y conversaciones fue registrada en un diario de campo. Para su análisis me serví de la perspectiva dialógica (Bajtín, 1982) ubicando y registrando los sujetos discursivos participantes en las prescripciones alimentarias dirigidas a la población residente en la zona de estudio. De cada uno de los sujetos, identifiqué las prácticas prescritas, las explicaciones y los argumentos subyacentes a estas, determinando las continuidades y rupturas argumentativas entre sí y con el discurso biomédico escrito.

\section{Área de estudio}

El barrio se asienta en un cerro en la zona sur del área metropolitana de Monterrey. El gris del concreto y los techos de lámina, que se mezclan con la maleza, denotan la precariedad de las viviendas predominantemente autoconstruidas. De acuerdo con información oficial (Inegi, 2010), el barrio tiene 2 mil 600 habitantes, de los cuales el 50\% son mujeres (1.289) y el $23 \%$ son menores de entre o y 11 años (615). Según la misma fuente, el 9o\% de la población cuenta con los servicios básicos (agua, luz, gas y drenaje), aunque he podido constatar que el suministro es problemático. Por ejemplo, la provisión del agua potable en la parte superior del cerro es únicamente durante veinte minutos al día. El 10o\% de la población cuenta con cobertura de salud. Las ocupaciones predominantes se ubican en la construcción, atendiendo pequeños comercios y en el trabajo doméstico (en el caso de las mujeres). Las condiciones de trabajo pueden ser caracterizadas como precarias: bajos salarios (alrededor de USD 270 al mes) y poca estabilidad laboral. El barrio cuenta con cinco planteles escolares, desde preescolar hasta bachillerato. Existe una decena de pequeños expendios de alimentos en los que predomina la comida chatarra y únicamente en tres de ellos se ofertan frutas, verduras y hortalizas. Aunque algunas viviendas cuentan con árboles frutales, no existen huertos urbanos. 


\section{Resultados}

Según la información obtenida mediante la encuesta, el medio de comunicación mayormente utilizado es la televisión (95\%), seguida de redes sociales como Facebook (72\%), WhatsApp (68.3\%) y YouTube (56.7\%). El 9o\% de las personas encuestadas expresó haber recibido información de salud y el $75 \%$ sobre alimentación a través de esos medios. La comida chatarra ( $43 \%$ ) y el refresco (56\%) se consumen de manera considerable, pese a que la reputación de estos productos es negativa, en opinión del $55 \%$ y el $72 \%$ de las personas, respectivamente.

\section{Sujetos, dispositivos y discursos}

\section{Médicos}

A partir de la interacción con estos sujetos discursivos, la población se encuentra familiarizada con restricciones alimentarias generales, dirigidas sobre todo a personas diagnosticadas con alguna enfermedad asociada a la alimentación, particularmente diabetes. Estas restricciones fueron señaladas en los talleres. Las mujeres frecuentemente hacen alusión a que "pecan" o han comido "veneno" cuando se refieren a ciertos alimentos que se hallan "prohibidos" por los médicos, como pan, tortillas de harina, carne de res o café. Lo prohibido es asociado fuertemente al placer y su restricción implica un acto de autocontrol: "Lo que hace daño es lo más sabroso", señaló una participante.

Durante la consulta de una residente de la colonia a la que acompañé, el médico sentenció:

Otra de las complicaciones muy usuales en la diabetes, que frecuentemente da a personas que presentan sobrepeso u obesidad, es que con el tiempo van perdiendo la vista, o en el peor de los casos, ya muy avanzados, que no se cuidaron nunca, se dañan los riñones...en cuanto a qué alimentos comer o no comer, todo lo que son carbohidratos y grasas. (Diario de campo)

A diferencia de otros estudios (Théodore et al., 2011), que señalan la generación de categorías opuestas entre prácticas saludables y no saludables, en el caso que presento se ha generado un orden de clasificación alimentaria sobre aquello prohibido o permitido. Esto debido a la asociación entre las prescripciones propuestas con situaciones patológicas.

Esto ha producido tres situaciones: en primer lugar, dichas prohibiciones, al ser contradictorias con las prácticas cotidianas, son llevadas a cabo de manera episódica al ser relacionadas con enfermedades o descontroles corporales. En segundo lugar, al ser asociadas con situaciones patológicas o anormales, las prescripciones han sido identificadas con población adulta o enferma, lo que tiende a excluir de ellas a los niños, por considerarles una población sana. En tercer lugar, este orden de clasificación entra en contradicción con las propuestas elaboradas por otros sujetos discursivos, como detallaré a continuación.

\section{Nutriólogos y promotores de salud}

Estos sujetos proponen un discurso centrado en prescripciones alimentarias denominadas "saludables" y de carácter permanente, en oposición al discurso médico, episódico y estructurado en la prohibición y patologización. A través de la interacción con nutriólogos y promotores de salud, la población se ha familiarizado con ciertos temas como el "plato del buen comer" y con significaciones como la asociación entre "energía" y azúcar, como queda de manifiesto en la charla sobre "estilos de vida saludables" impartida por una nutrióloga en un centro de nutrición privado:

El carbohidrato lo que nos aporta es energía, solamente el carbohidrato nos da energía. Es por eso que no podemos prescindir de él. Y el cerebro únicamente come carbohidratos, el cerebro no come ni proteínas ni come grasas, es decir, si ustedes tienen mucha hambre y se comen un pedazo de carne va a seguir dando lata porque la carne llenó tu estómago, pero el cerebro no necesita la proteína, no te hace nada porque necesita azúcar... (Diario de campo)

A diferencia de los nutriólogos, que atienden principalmente en consultas individuales, la educación para la salud constituye la actividad sustantiva de los promotores. En su mayoría trabajadores sociales, aunque reproducen el discurso de los estilos de vida, no reciben formación biomédica e incluso en el caso de Nuevo León su programa de estudios no incluye ninguna asignatura relacionada con la salud. La 
información que transmiten se basa en distintos manuales otorgados por las autoridades que ellos "traducen” y “adaptan” a la población.

La circulación de dicha información se realiza siguiendo un orden jerárquico institucional, similar a lo que ha reportado Briggs (2003). El contenido temático se decide en el nivel central que corresponde a la Federación, posteriormente es recibido en la Dirección de Promoción de la Salud estatal y, a partir de ahí, es replicado por promotores de salud de distintas jerarquías hasta llegar a los operativos, quienes reproducen la información a la población, auxiliados por promotores "voluntarios", residentes de las poblaciones destinatarias de la información.

Esta forma de reproducción discursiva provoca que se generen continuas resignificaciones durante la circulación del discurso y, por lo tanto, la información que recibe la población es similar al sentido común local, con una tendencia a desestimar la obesidad infantil.

A continuación, presento un fragmento de una conversación sostenida con una promotora en un centro de salud al que acudí, simulando ser padre de un niño obeso en búsqueda de charlas educativas.

Si no tiene actividad física, si está viendo mucho la tele, esos son los factores que hacen que se haga el sobrepeso -sonrió, hizo un gesto de despreocupación y siguió hablando-, pero está chiquito, todavía se recupera. No te preocupes. Cuando trabajan los papis y los dejan con los abuelitos -la promotora hizo una expresión, fingiendo que se dirigía a un niño-: “¿qué quiere comer mi chiquito?". ¿A poco no? Luego lo más grasoso es lo más sabroso. ¿A poco no? [Risas]. (Diario de campo)

\section{Programas gubernamentales}

El contacto con la institución médica se intensificó con el programa gubernamental Prospera ${ }^{2}$, que consistía en realizar transferencias monetarias condicionadas. Una de estas condiciones era la asistencia a "charlas" de prevención, dentro de las que se incluía la promoción de "estilos de vida saludables”.
Junto con este programa, la población interactuó con nutriólogos y promotores de salud, sujetos discursivos que, como expuse, estructuran un discurso distinto al de los médicos. El programa desapareció a principios de 2019, por lo que la población dejó de asistir a las charlas y, por tanto, las acciones de educación en salud se centraron en la consulta individual o en las escuelas.

Los planteles escolares constituyen un dispositivo importante para introducir el discurso de los estilos de vida a la colonia a través del programa nacional "Salud en tu Escuela”. Como parte de sus líneas de acción se establece la promoción de "hábitos saludables y cambios de comportamiento" (México, 2017), mediante charlas dirigidas a escolares acerca de la nutrición, impartidas por promotores de la Secretaría de Salud que acuden una vez por mes a las escuelas.

De acuerdo con una conversación sostenida con el titular de la Dirección de Prevención del Delito, esta dependencia también ha incorporado a sus acciones la promoción de "estilos de vida saludables" a partir de una perspectiva amplia de la prevención y de la seguridad. Con este fin se ha gestionado la entrada a la colonia de interventores como nutriólogos y de otros agentes que, como en mi caso y con intereses disímiles, reproducimos un discurso similar. La Dirección de Prevención del Delito ha utilizado la intervención más bien con fines de interpelación y acercamiento a la población, considerando la alta incidencia delictiva en la zona, como sostuvo un policía del programa "Regios Trabajando".

El discurso de los "estilos de vida" se ha focalizado en la puesta en práctica de acciones individuales (Menéndez; Di Pardo, 2009) que, sin reparar en las condiciones materiales de la población, reproduce un discurso moralizante que responsabiliza a los sujetos y tiende a proponer prácticas ideales que son insostenibles en el mediano plazo, lo que incentiva el carácter episódico en la adopción de las prácticas prescritas.

\section{Medicinas alternativas}

En mis recorridos por el barrio identifiqué la existencia de tres espacios en los que empresas

\footnotetext{
2 Prospera fue un programa gubernamental dirigido a atender pobreza, salud y educación de la población mexicana, con vigencia de 1995 a 2018.
} 
privadas organizan reuniones en las que se proponen prácticas de medicalización centradas en el consumo de suplementos alimenticios. De acuerdo con su discurso, los productos ofertados, elaborados a partir de "conocimientos científicos", proporcionan mejores aportes nutricionales que los alimentos considerados saludables.

Durante mi asistencia a uno de estos espacios, la "promotora", en cuya casa se había organizado una charla de nutrición, mencionaba que al consumir una de las malteadas elaboradas por la empresa se podían obtener muchos más nutrientes que al ingerir cualquier alimento, de manera que tomar una malteada en la mañana y otra en la noche era suficiente para "nutrirse". Otro elemento central de este discurso radica en su concepción de lo "natural" y de la "pérdida" de nutrientes que conlleva el proceso de distribución y almacenamiento de productos. Esa carencia general de nutrientes es la principal justificación para que las personas compren sus vitaminas y productos.

Desafortunadamente, vivimos en un mundo en el que todo está procesado. La carne que comemos está llena de hormonas. ¿Ustedes comen verduras, verdad? Se cuidan. ¿Comen ensaladas y frutas? Está muy bien -sonríe-. Si viviéramos en el campo y cosecháramos nuestras manzanas, nuestras acelgas, estaría muy bien. Pero no. La manzana que hoy te comiste -dice y señala a un asistente-, estuvo meses congelada. Ya no tiene nutrientes... ¿Por qué creen que hay tanta obesidad? No tenemos nutrientes que ayuden a nuestro metabolismo... (Diario de campo)

A diferencia de los tipos anteriores de medicalización, que prescriben prácticas, restrictivas o selectivas, el discurso de las medicinas alternativas propone un consumo de medicamentos que genera un tipo de medicalización mercantilizado denominado “medicamentación” (Williams; Gabe; Martin, 2012). Este proceso ha incentivado prácticas de sustitución que, como discutiré posteriormente, son relevantes para comprender las "prácticas de refracción”.

Identifico, así, tres tipos de discursos alimentarios: al primero lo denomino "restrictivo". Consiste en disminuir o eliminar ciertos alimentos y aunque está asociado con los médicos, su lógica se manifiesta en distintos tipos de “dietas”. El segundo, de carácter “selectivo", propone discriminar los alimentos según su identificación con lo saludable. El tercero corresponde al orden de la "medicamentación", a través de productos que "prometen" mejor salud a través de su consumo.

\section{Medios de comunicación}

En los medios de comunicación circulan simultáneamente los tres discursos mencionados. Durante un taller, la discusión se centró en “dietas” que las mujeres siguen cotidianamente. Nombraron varias que habían conocido en Facebook o que se compartían vía WhatsApp, como la dieta "keto", que consiste en consumir solo proteínas. También señalaron algunas recomendaciones asociadas al discurso selectivo, por ejemplo, comer primero ensalada antes que cualquier platillo. Por supuesto, abundan los anuncios de medicamentos para "bajar de peso".

En los canales de televisión más vistos por la población encuestada (Multimedios y Televisa) aparece con frecuencia un producto llamado "Sabifrut", anunciado como "una bebida hecha con sábila de verdad".

La primera toma del promocional encuadra a una mujer, presumiblemente madre de dos niñas sonrientes. Su voz en off explica el proceso de producción de la mercancía a partir de imágenes propias de un contexto rural. La mujer enuncia la frase "es bueno para mi familia", acompañada por la imagen de un médico, quien enlista los componentes de la bebida y sus beneficios.

La utilización de símbolos asociados a la salud, como lo natural, y la figura de autoridad del médico es frecuentemente usada por la industria de la reducción de daño, como Nichter (2006) la ha denominado. Esta estrategia discursiva ha sido bastante efectiva. De acuerdo con la encuesta realizada, "Sabifrut" es una de las bebidas azucaradas más consumidas por los niños de la comunidad estudiada.

\section{Conocimiento autorizado}

Un elemento fundamental para comprender las razones por las que la población acepta la información recibida es la configuración de dicha 
información como "conocimiento autorizado" (Browner; Press, 1996), que, según los autores citados, es un cuerpo de saberes normado, con un alto grado de formalización, pero sobre todo con mayor legitimidad que otros conocimientos por la efectividad que se le atribuye y por la posición de poder de origen. Como expuse anteriormente, el discurso biomédico constituye entre la población de estudio un conocimiento autorizado.

Los medios de comunicación conforman igualmente un campo de conocimiento autorizado. Contrario a lo que señalan Browner y Press (1996), considero que la norma no es indispensable para configurar un conocimiento autorizado, como sí lo es su capacidad para orientar comportamientos.

Durante los talleres, me percaté de que las mujeres habían desarrollado ciertas prácticas, como evitar determinados productos, basadas en la información compartida en Facebook o WhatsApp. Por ejemplo, una de ellas evita comprar y consumir lácteos, luego de que se difundió en redes sociales la noticia de que estaban asociados con el cáncer. A partir de una razón similar, en el grupo de "minichef" se evita comer embutidos.

No obstante la autoridad de los diversos sujetos discursivos, la población frecuentemente pone en duda sus prescripciones. Por ejemplo, en una ocasión una mujer del barrio me comentó que el diagnóstico de obesidad infantil de su hijo, realizado por una médica durante la consulta, se debía más bien a que esta profesional era "demasiado flaca".

La diversidad de sujetos y dispositivos que prescriben prácticas alimentarias da origen a una reproducción discursiva contradictoria. De acuerdo con mis observaciones, la población ha desarrollado una estrategia para lidiar con estas contradicciones: elige aquellos fragmentos de información que son congruentes con modelos explicativos practicados en su vida cotidiana. En este sentido, la información se "refracta" a partir de una discriminación discursiva.

\section{Refracción}

Una de las mujeres que anteriormente había mencionado que le gustaban las ensaladas comentó que consideraba que era sano comer vegetales verdes haciendo alusión a que "verde" era "natural".
Posteriormente, agregó que durante sus consultas le habían explicado que tenía que comer productos naturales. Esta participación fue seguida por otra asistente que dijo que debido a que provenían de un rancho, para ella lo sano era lo más natural, ya que no estaba contaminado. En la vida rural todo era más sano, ya que provenía directamente de la naturaleza. (Diario de campo)

La participante arriba mencionada ha valorado clara y positivamente la prescripción médica, ya que esta coincide en su totalidad con la asociación establecida en forma previa entre lo natural y lo sano. Esta asociación semántica es reproducida con frecuencia en las redes sociales, particularmente a través de la preocupación manifestada por diferentes usuarios sobre los alimentos procesados, especialmente usuarios que, a la vez, son sujetos adscritos a instituciones médicas.

Esta oposición entre lo natural y procesado recuerda el trabajo de Mary Douglas (2003), en el sentido de que lo artificial, lo que se añade, contamina, configurando una estructura de ordenamiento de significados que permite distinguir aquellos alimentos permitidos o deseables de aquellos que deben restringirse. Este ordenamiento ha sido bien aprovechado por "la industria de la reducción de daño" para anunciar productos alimenticios.

Así como Douglas (2003), considero que las constantes contradicciones informativas inherentes a la polifonía generan mensajes ambiguos. En la medida en que no pueden ser fácilmente incorporados al orden establecido en la estructura de significados, constituyen espacios abiertos para resignificaciones y por lo tanto para refracciones.

Desde esta perspectiva, es posible plantear que parte del persistente consumo del refresco, particularmente de la Coca-Cola, se explica como producto de una refracción de la asociación semántica entre azúcar y energía reproducida especialmente por los sujetos pertenecientes a las instituciones médicas. Estas personas se refieren al significado biomédico de energía como una capacidad metabólica indispensable para la vida. En cambio, para la población "energía” tiene connotaciones asociadas con sensaciones corporales y anímicas. Durante el trabajo de campo, presencié en diferentes ocasiones que las personas toman Coca-Cola cuando "necesitan energía" o se les 
“baja la presión”. En esta lógica, a los niños se les da a tomar esta bebida cuando los notan "débiles" o "tristes".

Por supuesto, como he señalado antes, las posibilidades materiales son elementos importantes de la refracción. Durante una conversación, una de las asistentes al taller me comentó que ella seguía las recomendaciones de la nutrióloga hasta donde le era posible en términos económicos. Después de esto, simplemente "compro lo que me alcanza", afirmó. Al despedirnos, me enseñó un recipiente que contenía avena en remojo, señalando que era lo que le quedaba y que tal vez no le alcanzaría para completar la prescripción de un recetario adquirido vía WhatsApp, en el que se incluía el consumo de avena cinco veces por semana.

Otros factores determinantes de la refracción corresponden a elementos pragmáticos como el tiempo. En otra ocasión, la plática con otra de las participantes del taller fue interrumpida por el llanto de su nieto. La mujer se levantó y sacó del refrigerador un envase de plástico, destapó el producto y se lo acercó al bebé, quien inmediatamente lo tomó como si fuera una mamila. La señora me indicó que era una papilla de manzana, mostrándome con satisfacción que era baja en azúcar. Posteriormente, explicó que, para ella, que tenía que levantarse desde muy temprano, hacer el desayuno para sus hijos y esposo, ir a trabajar como empleada doméstica, regresar a preparar la comida y después cuidar a su nieto, era muy útil encontrar productos prácticos que le ahorraran tiempo.

Detrás de este pragmatismo se encuentran condiciones materiales precarias, acentuadas por una feminización de los cuidados, que en este caso recae en las abuelas, quienes ayudan a sus hijas a cuidar de los niños mientras ellas cumplen sus horarios laborales.

La refracción discursiva genera diversas prácticas cuyo propósito manifiesta una preocupación por proteger la salud de los niños. En una de ellas, a la que denomino "práctica de sustitución", un producto considerado como dañino es reemplazado por otro, como en el caso de adultos que consumen productos light. En el caso de los niños, el refresco ha comenzado a ser sustituido por jugos o por bebidas azucaradas que son anunciadas como productos naturales. Un ejemplo de ello, como ya se mencionó, es el consumo de "Sabifrut".
Otra práctica común en la colonia es administrar los alimentos de acuerdo con un horario. Por ejemplo, a los niños se les restringe el consumo de comida chatarra, aunque esta sea permitida durante los fines de semana o en fiestas. Estas prácticas, que denomino de "restricción", intentan disminuir el daño de un producto a partir del control de su ingesta.

Durante una comida en casa de una familia, al repartir el refresco, la abuela se sirvió únicamente la mitad del vaso, llenando la mitad restante con agua. Cuando pregunté los motivos de ese comportamiento, la abuela contestó que tomar "demasiado" refresco era dañino. Ante mi pregunta sobre la razón por la que el mismo criterio no había aplicado a los niños, comentó que ellos no estaban enfermos. Una vecina invitada a la comida comentó que ella no les daba refresco a sus hijos, ya que prefería darles bebidas que había visto por Facebook que "purificaban la sangre”. De acuerdo con algunos videos que me mostró, se trataba de productos con sábila cuyo efecto purificador era explícitamente señalado. (Diario de campo)

Los niños son excluidos de algunas medidas de restricción, pero al mismo tiempo son sujetos de prácticas cuya justificación es procurarles una mejor salud. Es decir, la evitación de un daño no es el único elemento en juego. Durante una charla para personas diabéticas en un centro de salud, la nutrióloga indicó que para atender la hipoglucemia era conveniente comer alguna manzana, aunque el efecto era mayor si estas eran hervidas. Una de las asistentes, sentada detrás de mí, comentó que a partir de ese momento le daría manzanas hervidas a su hijo.

El deseo por la salud de los infantes incentiva de una manera más importante prácticas alimentarias que aquellas dirigidas a evitar un daño, aunque muchas de estas, como se ha explicado, han sido refractadas por la población.

\section{Discusión y conclusiones}

A partir de los sujetos discursivos y dispositivos identificados en la prescripción de prácticas alimentarias en el área de estudio, he reconocido la presencia de tres tipos de discursos: restrictivo, selectivo y aquel asociado a la medicamentación. 
Estos hallazgos demuestran el carácter polifónico y contradictorio en las prescripciones alimentarias que cotidianamente recibe la población. Las discrepancias discursivas son articuladas e insertadas en una estructura material y de significación a partir de un procesos que denomino "refracción", generando prácticas alimentarias que son incomprensibles o incluso indeseables para las autoridades sanitarias. Esto podría explicar los pobres resultados obtenidos para la adopción de prácticas saludables, particularmente en los niños, desde la perspectiva de estas autoridades.

Pese a sus contradicciones, estos discursos presentan cuatro elementos en común: (1) son de naturaleza prescriptiva, (2) individualizan el problema, (3) explican la situación a través de una concepción biológica, haciendo énfasis en lo "natural”, y (4) apelan a una figura de autoridad, particularmente médica. Estos elementos constituyen la estructura de un género discursivo biomédico, lo cual puntualiza su carácter dominante y resalta la polifonía de los procesos de medicalización en los colectivos sociales. Por otro lado, confirma la vinculación que existe entre el discurso biomédico nutricional y la industria alimentaria, tal como lo señala Marion Nestle (2018).

Otra contribución consiste en mostrar la autoridad que los medios de comunicación y redes sociales tienen en la producción de prácticas alimentarias, circunstancia que debería incorporarse en los estudios de los procesos de medicalización, considerando que la poca incursión de las instancias públicas en estas tecnologías ha dejado al mercado un nicho de comunicación para la salud. En este sentido, existe suficiente evidencia en la literatura que demuestra la efectividad en el empleo de los medios electrónicos para la modificación de prácticas alimentarias (Gamboa-Delgado; Izeta; Amaya-Castellanos, 2018). No obstante, estas estrategias tienden a considerar a los sujetos como receptores pasivos de información, particularmente a los niños (Equipo Editorial, 2013), aspecto que el presente trabajo cuestiona y que debería ser tomado en cuenta en el diseño de políticas de salud.

Respecto a los límites de la medicalización, diversos estudios han sugerido que las contradicciones entre la información de salud conocida por la población y sus prácticas pueden ser explicadas por una inadecuada percepción del riesgo (Hossain et al., 2019).
Sin embargo, a la luz de los hallazgos etnográficos de este trabajo es posible cuestionar tal aseveración. Creo que, efectivamente, la información proporcionada tiene límites importantes para inducir prácticas alimentarias, pero no por su contenido ni por su ineficacia para comunicar un riesgo, sino por el carácter polifónico y contradictorio de su reproducción.

\section{Referencias}

AGAMBEN, G. ¿Qué es un dispositivo? Sociológica, Ciudad de México, v. 26, n. 73, p. 249-264, 2011.

ARCAN, C. et al. Somali, Latino and Hmong parents' perceptions and approaches about raising healthy-weight children: a communitybased participatory research study. Public Health Nutrition, Cambridge, v. 21, n. 6, p. 1079-1093, 2018.

BAJTÍN, M. M. Estética de la creación verbal. Ciudad de México: Siglo XXI, 1982.

BATIS, C. et al. First-year evaluation of Mexico's tax on nonessential energy-dense foods: an observational study. PLoS Medicine, San Francisco, v. 13, n. 7, art. e1002057, 2016.

BRIGGS, C. L. Why nation-states and journalists can't teach people to be healthy: power and pragmatic miscalculation in public discourses on health. Medical Anthropology Quarterly, Washington, DC, v. 17, n. 3, p. 287-321, 2003.

BROWNER, C. H.; PRESS, N. The production of authoritative knowledge in American prenatal care. Medical Anthropology Quarterly, Washington, DC, v. 10, n. 2, p. 141-156, 1996.

CARPENTER, D. M. et al. Conflicting health information: a critical research need. Health Expectations, Oxford, v. 9, n. 6, p. 1173-1182, 2016.

CONRAD, P. Medicalization and social control. Annual Review of Sociology, Palo Alto, v. 18, p. 209-232, 1992.

DOMÍNGUEZ, M. C. M. La medicalización y la autonomía: etnografía de experiencias maternas en Chiapas. Tese (Doutorado em Antropologia Social) - Centro de Investigaciones y Estudios Superiores en Antropología Social, San Cristóbal de las Casas, 2019. 
DOUGLAS, P. M. Purity and danger: an analysis of concepts of pollution and taboo. Abingdon: Routledge, 2003.

DREWNOWSKI, A. The economics of food choice behavior: why poverty and obesity are linked. Nestlé Nutrition Institute Workshop Series, Basel, v. 73, p. 95-112, 2012.

EQUIPO EDITORIAL. La alimentación infantil, el marketing y los medios de comunicación. Pediatría Atención Primaria, Madrid, v. 15, n. 6o, p. 299-303, 2013.

GAMBOA-DELGADO, E.; IZETA, E. E.; AMAYACASTELLANOS, A. Aplicabilidad de las teorías de comunicación en salud en el campo de la educación nutricional. Revista Chilena de Nutrición, Santiago, v. 45, n. 1, p. 6o-64, 2018.

GÓMEZ, A. P.; DÍAZ, R. R. ¿La información sobre salud está condicionada por la nacionalidad de los enfermos? El ébola como estudio de caso. Saúde e Sociedade, São Paulo, v. 28, n. 3, p. 253-266, 2019.

GUBER, R. La etnografía: método, campo y reflexividad. Bogotá: Norma, 2001.

HOSSAIN, M. S. et al. Is childhood overweight/ obesity perceived as a health problem by mothers of preschool aged children in Bangladesh? A community level cross-sectional study. International Journal of Environmental Research and Public Health, Basel, v. 16, n. 2, art. 202, 2019.

INEGI - INSTITUTO NACIONAL DE ESTATÍSTICA, GEOGRAFÍA E INFORMÁTICA. Censo de población y vivienda 2010. Aguascalientes, 2010. Disponível em: <http://bit.ly/36zSqD4>. Acesso em: 28 nov. 2019.

INSP - INSTITUTO NACIONAL DE SALUD PÚBLICA; SSA - SECRETARÍA DE SALUD FEDERAL. Encuesta nacional de salud y nutrición de medio camino 2016: informe final de resultados. Cuernavaca, 2016.

LOVEMAN, E. et al. Parent-only interventions for childhood overweight or obesity in children aged 5 to 11 years. Cochrane Database of Systematic Reviews, Oxford, n. 12, art. CDo12008, 2015.

MENÉNDEZ, E. L.; DI PARDO, R. B. Miedos, riesgos e inseguridades: los medios, los profesionales y los intelectuales en la construcción social de la salud como catástrofe. Ciudad de México: Ciesas, 2009.
MENESES-NAVARRO, S.; MELÉNDEZ-NAVARRO, D.; MEZA-PALMEROS, A. Contraceptive counseling and family planning services in the Chiapas highlands: challenges and opportunities for improving access for the indigenous population. In: SCHWARTZ, D. A. (Ed.). Maternal death and pregnancyrelated morbidity among indigenous women of Mexico and Central America: an anthropological, epidemiological, and biomedical approach. New York: Springer, 2018. p. 271-300.

MÉXICO. Secretaría de Educación Pública; Secretaría de Salud. Modelo educativo: salud en tu escuela. Ciudad de México, 2017. Disponível em: <http://bit.ly/2YFsIdm>. Acesso em: 29 nov. 2019.

NESTLE, M. Unsavory truth: how food companies skew the science of what we eat. New York: Basic Books, 2018.

NICHTER, M. Reducción del daño: una preocupación central para la antropología médica. Desacatos, Ciudad de México, n. 20, p. 109-132, 2006.

ROSE, N. Beyond medicalisation. The Lancet, Amsterdam, v. 369, n. 9562, p. 700-702, 2007.

ROSEN, G. De la policía médica a la medicina social: ensayos sobre la historia de la atención a la salud. Ciudad de México: Siglo XXI, 1985.

SAFDIE, M. et al. An ecological and theoretical deconstruction of a school-based obesity prevention program in Mexico. International Journal of Behavioral Nutrition and Physical Activity, London, v. 11, art. 103, 2014.

THÉODORE, F. L. et al. Representaciones sociales relacionadas con la alimentación escolar: el caso de las escuelas públicas de la Ciudad de México. Salud Colectiva, Buenos Aires, v. 7, n. 2, p. 215-229, 2011.

VOLÓSHINOV, V. N. El marxismo y la filosofía del lenguaje. Buenos Aires: Godot, 2009.

WILLIAMS, S.; GABE, J.; MARTIN, P. Medicalization and pharmaceuticalization at the intersections: a commentary on Bell and Figert (2012). Social Science \& Medicine, Oxford, v. 75, n. 12, p. 2129-2130, 2012.

Recibido: 29/07/2020

Aprobado: 09/09/2020 